\title{
Influence of injection mode and antioxidant dietary on the toxicity of Doxorubicin as a chemotherapy drug
}

\begin{abstract}
Doxorubicin (Dox), among others, is a chemotherapy drug, used to treat different types of cancers. It considered as potent anticancer therapeutics with significant effectiveness in lymphomas and many solid tumors. Patients with breast cancer, in particular, doxorubicin are the first choices of therapy. Unfortunately, the clinical use of Dox is restricted by its ability to develop cardiomyopathy and congestive heart failure. Administration route of Doxorubicin recommended of being intravenous. However, three other administration routes also are being applied depending on the case and to avoid faster cardio toxicity development. Development of cardiomyopathy, due to the prolong Doxorubicin injection that leads to sharp rises in left ventricular dysfunction followed by other complications, gave an attention to a relentless search for the better anthracycline with less cardio toxicity. Few studies were carried out to decrease the side effect of this drug, among these was choosing the mode of its injection or use antioxidant dietary in combination and modifying their mode of action. Comparison of different injection routes of Dox and their side effects were our main intention. The capability of using herbal medicine, have antioxidant property or protective effect like those of Moringa seeds, were also considered.
\end{abstract}

Keywords: doxorubicin, injection route, anticancer drug acclimatized
Volume 9 Issue 6 - 2018

\author{
Heba N Gad EL-Hak,' Abdelraouf A \\ Moustafa, ${ }^{2}$ Samira R Mansour ${ }^{3}$ \\ 'Lecturer of cell biology and histology, Department of Zoology, \\ Suez Canal University, Egypt \\ ${ }^{2}$ Professor of plant ecology, Department of Botany, Science Suez \\ Canal University, Egypt \\ ${ }^{3}$ Professor of Microbiology, Department of Botany, Science Suez \\ Canal University, Egypt
}

Correspondence: Samira R Mansour, Professor of Microbiology, Botany Department, Faculty of Science, Suez Canal University, Ismailia, Egypt, Tel +201003686076, Fax +20 643230416,Email samirarmensour@hotmail.com

Received: October 28, 2018 | Published: November 21, 2018

\section{Introduction}

Doxorubicin (Adriamycin, trade name) is one of the most frequently used drugs for cancer chemotherapy. This drug proved to be the most effective as systemic chemotherapy, particularly for liver cancers. ${ }^{1}$ Dox is an anthracycline antibiotic, a class of drug, which is produced naturally by actinobacteria of genus Streptomyces named S.peucetius. It consists of a naphthacenequinone linked through a glycosidic bond at ring atom 7 to an amino sugar named daunosamine. Dox is considered one of two drugs (daunorubicin) of the first generation of anthracycline antibiotics. ${ }^{2}$ This drug has Antineoplastic activity. ${ }^{3}$ According to the Biopharmaceutical Classification System, Dox belongs to class III that is characterized by high solubility and low permeability. ${ }^{4}$ So, it is preferable to be administrated intravascular.

The mode of action of Dox is intercalated between base pairs in the DNA helix, thereby preventing DNA replication and ultimately inhibiting protein synthesis. The mechanism of inhibition is due to the involvement of enzyme blocking named Topoisomerase. ${ }^{5}$ Experimental evidence indicated that DNA intercalation triggers the cleavage of DNA by Topoisomerase-II, yielding serious disturbances in the tertiary structure of DNA. As a result, it slows down or stops the growth of cancer cells where rapid and uncontrolled cell division has occurred. In general, normal cells, during the cell cycle, will stop dividing due to checkpoints in which two periods are recognized: rest and active growing and division periods. The checkpoints ensure that the cells are not growing too fast and do have any mistakes. In the case of cancer, the cells do not have these checkpoints and are not limited in how large or how fast they grow. Chemotherapy agents like Doxorubicin inhibit cell division by binding to DNA within the cell, causing damage that prevents the DNA from replicating of the cancer cell. When cancer cells are dividing, they need Topoisomerase enzyme to replicate their DNA; when it is blocked, DNA damage occurs and the cell will stop dividing and growth will slow down. ${ }^{6}$
In addition, in exploring heart toxicity-side effect, some studies proved that Dox causes dose-dependent cardio toxicity. This may lead to irreversible heart failure by different mechanisms. ${ }^{7}$ Among these mechanisms is a progressive reduction of left ventricular function that leads to a decrease in fractional shortening and ejection fraction. Therefore, patients during the course of Dox therapy exposed to the continuous rhythm that followed by life-threatening congestive heart failure. ${ }^{8}$ Mean while, production of reactive oxygen species, calcium imbalance, mitochondrial damage, and apoptosis are also considered in Dox cardio toxicity condition. ${ }^{9}$

Clinically, Dox chemotherapy drug is mainly administered intravenously, as mentioned above, but depending on the type of cancer and the therapeutic indication, as in solid tumors or acute leukemias, different administration routes were followed..$^{10,11}$ Therefore, four routes of Dox administration have been used: Intravenous, Intravesical, Intra-arterial, and Intraperitoneal. Intravenous administration of Doxorubicin should be performed with caution due to its direct effect on the heart that causes cardiomyopathy and congestive heart failure. ${ }^{12,13}$ Meanwhile, the Intravesical administration is applied for the treatment of superficial bladder tumors or as prophylaxis to reduce recurrence after transurethral resection. ${ }^{14,15}$ A major advantage of Intravesical Dox administration is giving chemotherapy directly into the bladder instead of injecting it into the bloodstream. Accordingly, it is a direct contact of the chemotherapy to cancer tissue without being reaches to other parts of the body. This route of administration helps people to avoid many of the side effects that can occur with intravenous administration of Dox. However, the main side effects that can occur in Intravesical Dox administration are irritation and a burning feeling in the bladder. ${ }^{16}$

The third route of Doxorubicin administration is through intraarterial, particularly for patients with hepatocellular carcinoma (HCC), to reduce its toxicity and produce intense local activity. For 
HCC patients, systemic administration of Dox showed limited clinical benefits where the accumulative dose of Dox could lead to cardio toxicity and high rate of mortality. ${ }^{17}$ However, when the drug was given by the hepatic artery-route, tumor shrinkage was recorded and partial responses were seen in $30-70 \%$ of patients. ${ }^{18}$ Therefore, the development of more effective Dox to achieve a higher percentage of HCC-patient recovery, future directions for using this drug in therapy, is intensively recorded ongoing researches.

Although Doxorubicin has hazard side, fourth route of Dox through intraperitoneal administration, is used by some researches in their experimental animal. ${ }^{19,20}$ In the meantime, this route of administration is preferable due to the majority of the injected Dox-dose remained in the abdominal cavity without being incorporated into the systemic circulation which can cause cardiomyopathy and congestive heart failure. ${ }^{21}$ However, the application of Dox Intraproteinal, local toxic effects must be controlled and more attention should be followed in order to facilitate clinical utility.

No comparative studies were done to explore the side effect of the different administration routes of Dox. However, a study done by Li \& Tang $^{22}$ showed that the pharmacokinetics of Doxorubicin in nude mice subjected to intravenous and intraperitoneal administration of specific dose given as a single shot $(12 \mathrm{mg} / \mathrm{kg})$ showed that the Dox concentration curves in kidney, heart, and striated muscle following intraperitoneal administration were approximately half the concentration curves following intravenous injection. However, in plasma and liver, Dox concentration curve showed nearly identical concentrations after $2 \mathrm{~h}$ distribution. Meanwhile, evaluation of Dox $\mathrm{LD}_{50}$ showed a significantly higher value after intravenous administration than those of intraperitoneal administration. Therefore, the administration route of Dox is still dependent on cancer types and the way to be delivered to tumor cells. However, more researches, right now, are focusing on how to improve delivery of the drug and decrease its toxicity.

\section{Common side effects of doxorubicin}

Common symptoms recognized as side effects after Dox delivered to the patient's body, by any routes mentioned, are: nausea associated with vomiting (may be severe), diarrhea, loss of appetite, missed menstrual periods, darkening of skin or nails, bruising, weakness, tiredness, eye redness, or puffy eyelids, abnormal heartbeat and stomach pain. ${ }^{23}$ Cumulative doses of Doxorubicin may give a reddish color to urine, tears, and sweat that may proceed for several days. ${ }^{24}$ Temporary hair loss may occur. However, after Dox treatment has ended, normal hair growth should return. ${ }^{25}$ However, more severe side effects may occur, such as chest pain, sudden cough with foamy mucus or coughing up blood, urination less than usual or not at all or bloody urine, fever, chills, body aches, flu symptoms, sores in your mouth and throat; severe liver and heart problems.

\section{Improvement of doxorubicin toxicity}

Many studies are going forward to improve the side effect and toxicity of Doxorubicin. For that purpose, different therapeutic strategies for Dox administration were carried out which include construction of new generations of anthracycline; ${ }^{26}$ using stemcell-therapy $;{ }^{27}$ and searching for flavonoid compounds ${ }^{28}$ or active secondary metabolites of plants or microbes origin. ${ }^{29}$ Among these strategies, medicinal plants contain active compounds are a promising tool in concern for its natural products. In the meantime, nutritional components and antioxidant of some herbal medicine like those of Moringa species are also considered and being of potential uses ( Gad El-Hak et al 2017; Heba et al, unpublished data).

The bioavailability, metabolism, and biological activity of flavonoids compounds of plant origin rely upon their configuration, total number of hydroxyl groups, and substitution of functional groups about their nuclear structure. Many flavonoids are shown to have ant oxidative activity, free radical scavenging capacity, coronary heart disease prevention, hepato protective, anti-inflammatory, and anticancer activities, while some flavonoids exhibit potential antiviral activities. ${ }^{30}$ Therefore, using rich dietary regime has antioxidant and other nutrients supplement like those of Moringa seeds or leaves can be an alternative medicine to be used during chemotherapy medication. Moringa is a medicinal plant, now a days, it is used as a traditional remedy for many ailments. Moringa is a rich source of vitamins, minerals, and amino acids, it fights free radicals (the powerful antioxidant), inflammation and it helps in protecting the cardiovascular system, ${ }^{31}$ which is our main target for Dox medication. Meanwhile, Moringa supports brain health, protects the liver and contains antimicrobial and antibacterial properties. ${ }^{32,33}$ For all these properties of Moringa, we suggest to use its seeds or leaves along with Dox remediation to decrease its toxicity and cardiomyopathy.

\section{Conclusion}

The ability to protect against cardio toxicity, using natural dietary products, open a new area for enhancing clinical interest in exploiting modifications in the strategy of Doxorubicin medication as an anticancer drug. Using medicinal plant like Moringa is a promising therapeutic mediator to be used to reduce and fight congestive heart failure resulted from Doxorubicin toxicity. Since Moringa seeds or leaves have the high nutritional value that is considered as an excellent source of many vitamins (B2, B, A and C), some minerals and free radical scavenging capacity (antioxidant compounds), they can be taken along with Dox medication. Furthermore, the mentioned team of B vitamins has been found to reduce cardiomyopathy since they have an important role to reduce the level of homocysteine, a risk factor of heart disease. Accordingly, the antitumor therapy side effects may be acclimatized by taking dietary contains the promising medicinal Moringa plant, which may also help our own body to synthesize de novo (endogenous) antioxidants and balance shortage of $\mathrm{Ca}^{++}$that plays an important role in cell proliferation.

\section{Acknowledgments}

Authors express their deep thanks to all colleagues who encourage and help to finish up this minireview.

\section{Conflicts of interest}

Authors have no competing interests to declare.

\section{References}

1. Lammer J, Malagari K, Vogl T, et al. Prospective randomized study of doxorubicin-eluting-bead embolization in the treatment of hepatocellular carcinoma: results of the PRECISION V study. Cardiovasc Intervent Radiol. 2010;33(1):41-52.

2. Aubel-Sadron G, Londos-Gagliardi D. Daunorubicin and doxorubicin, anthracycline antibiotics, a physicochemical and biological review. Biochimie. 1984;66(5):333-352. 
3. Kurbacher CM, Wagner U, Kolster B, et al. Ascorbic acid (vitamin C) improves the antineoplastic activity of doxorubicin, cisplatin, and paclitaxel in human breast carcinoma cells in vitro. Cancer lett. 1996;103(2):183-189.

4. Varma MV, Khandavilli S, Ashokraj Y, et al. Biopharmaceutic classification system: a scientific framework for pharmacokinetic optimization in drug research. Curr Drug Metab. 2004;5(5):375-388.

5. Hande K. Etoposide: four decades of development of a topoisomerase II inhibitor. Eur J Cancer. 1998;34(10):1514-1521.

6. Tacar O, Sriamornsak P, Dass CR. Doxorubicin: an update on anticancer molecular action, toxicity and novel drug delivery systems. J Pharm Pharmacol. 2013;65(2):157-170.

7. Schwartz RG, McKenzie WB, Alexander J, et al. Congestive heart failure and left ventricular dysfunction complicating doxorubicin therapy: seven-year experience using serial radionuclide angiocardiography. $\mathrm{Am}$ J Med. 1987;82(6):1109-1118.

8. Barry E, Alvarez JA, Scully RE, et al. Anthracycline-induced cardiotoxicity: course, pathophysiology, prevention and management. Expert Opin Pharmacother. 2007;8(8):1039-1058.

9. Ferreira ALdA, Matsubara LS, Matsubara BB. Anthracycline-induced cardio toxicity. Cardiovascular \& Hematological Agents in Medicinal Chemistry. Formerly Current Medicinal Chemistry-Cardiovascular \& Hematological Agents. 2008;6(4):278-281.

10. Bonadonna G, Monfardini S, De Lena M, et al. Phase I and preliminary phase II evaluation of adriamycin (NSC 123127). Cancer Res. 1970;30(10):2572-2582.

11. Kubo T, Sugita T, Shimose S, et al. Targeted systemic chemotherapy using magnetic liposomes with incorporated adriamycin for osteosarcoma in hamsters. Int J Oncol. 2001;18(1):121-125.

12. Siveski-Iliskovic N, Hill M, Chow D, et al. Probucol protects against adriamycin cardiomyopathy without interfering with its antitumor effect. Circulation. 1995;91(1):10-15.

13. Baselli EC, Greenberg RE. Intravesical therapy for superficial bladder cancer. Oncology (Williston Park). 2000;14(5):719-729.

14. Herr HW, Laudone VP, Whitmore Jr WF. An overview of Intravesical therapy for superficial bladder tumors. $J$ Urology. 1987;138(6):1363-1368.

15. Manoharan M, Soloway MS. Optimal management of the T1G3 bladder cancer. Urol Clin North Am. 2005;32(2):133-145.

16. Pashos CL, Botteman MF, Laskin BL, et al. Bladder cancer. Cancer practice. 2002;10(6):311-322.

17. Kettenbach J, Stadler A, Katzler Iv, et al. Drug-loaded microspheres for the treatment of liver cancer: review of current results. Cardiovasc Intervent Radiol. 2008;31(3):468-476.

18. Tam K. The roles of doxorubicin in hepatocellular carcinoma. ADMET and DMPK. 2013;1(3):29-44.
19. Iliopoulos D, Hirsch HA, Struhl K. Metformin decreases the dose of chemotherapy for prolonging tumor remission in mouse xenografts involving multiple cancer cell types. Cancer Res. 2011;71(9):3196-3201.

20. Mukherjee S, Sau S, Madhuri D, et al. Green synthesis and characterization of mono dispersed gold nanoparticles: toxicity study, delivery of doxorubicin and its bio-distribution in mouse model. $J$ Biomed Nanotechnol. 2016;12(1):165-181.

21. Tahover E, Patil YP, Gabizon AA. Emerging delivery systems to reduce doxorubicin cardio toxicity and improve therapeutic index: focus on liposomes. Anticancer drugs. 2015;26(3):241-258.

22. Li M, Tang Z, Lv S, et al. Cisplatin cross linked $\mathrm{pH}$-sensitive nanoparticles for efficient delivery of doxorubicin. Biomaterials. 2014;35(12):3851-3864.

23. Carvalho C, Santos RX, Cardoso S, et al. Doxorubicin: the good, the bad and the ugly effect. Curr Med Chem. 2009;16(25):3267-3285.

24. Smith SD, Bergstrom SK, Zwerdling T. Toxicity of Antineoplastic Chemotherapy in Children. Drug Toxicity and Metabolism in Pediatrics. 2018. p. $185-220$.

25. Giuseppe G, Giulio FD, Morandini MP, et al. Scalp hypothermia in the prevention of doxorubicin-induced hair loss. Cancer Nurs. 1988;11(3):170-173.

26. Arcamone F, Animati F, Capranico G, et al. New developments in antitumor anthracyclines. Pharmacol Ther. 1997;76(1):117-124.

27. Sun TM, Wang YC, Wang F, et al. Cancer stem cell therapy using doxorubicin conjugated to gold nanoparticles via hydrazone bonds. Biomaterials. 2014;35(2):836-845.

28. Kaiserová H, Šimůnek T, van der Vijgh WJF, et al. Flavonoids as protectors against doxorubicin cardiotoxicity: Role of iron chelation, antioxidant activity and inhibition of carbonyl reductase. Biochim Biophys Acta. 2007;1772(9):1065-1074.

29. Demain AL. Pharmaceutically active secondary metabolites of microorganisms. Appl Microbiol Biotechnol. 1999;52(4):455-463.

30. Kumar S, Pandey AK. Chemistry and Biological Activities of Flavonoids: An Overview. Scientific World Journal. 2013;2013:162750.

31. Dhakar R, Maurya S, Pooniya B, et al. Maringa: The herbal gold to combat malnutrition. Chronicles of Young Scientists. 2011;2(3):119-125.

32. Coppin, Julia, Simon, James, Lavoie, Edmond, et al. A study of the nutritional and medicinal values of Moringa oleifera leaves from sub-Saharan Africa: Ghana, Rwanda, Senegal and Zambia. 2008. p. 103-113.

33. Ferreira PMP, Farias DF, Oliveira JTdA, et al. Moringa oleifera: bioactive compounds and nutritional potential. Revista de Nutrição. 2008;21(4):431-447. 